\title{
The Public Administrator: God? or Entrepreneur? Or Are They the Same in the Public Service?
}

\author{
A. R. Dobell \\ Institute for Research on Public Policy
}

\begin{abstract}
This paper distinguishes entrepreneurial behaviour in the public service (innovative activity where the risks and benefits are essentially personal or informed consent can reasonably be presumed) from decisions involving risks to others, usually anonymous and beyond consultation. It argues that decisions by public officials are more often of the latter type, imposing risks on others. This leads to a requirement for guidelines based first on rights and fundamental principles, then on a calculated risk-benefit analysis, and finally on a lively sense of personal responsibility. In the end, only leadership can instill a shared sense of organizational and personal values that assure the responsible exercise of administrative discretion in making risky collective decisions.
\end{abstract}

We all know that to innovate is great. But we also know that to err is human, and to forgive may be divine--but it is not customary. A tradition of entrepreneurship can only flourish in a culture in which the costs of error are not so great as to demand divine dispensation on a continuing basis.

To investigate the question of entrepreneurship in the public service, therefore, we must look to the corporate culture of the public service. Indeed, logically the analysis should start farther back with the culture of the nation itself. But a number of analytical questions also must be faced.

This paper sketches a few ideas about what might be seen as part of a very old debate in public administration, but one of the newest challenges in public organizations as well.

The paper briefly discusses the public servant as entrepreneur-- risk-taker on his or her own account, which involves delegation, decentralization, devolution of authority (all within some general policies or guidelines, presumably). Then it shifts to more extensive discussion of the public servant as God--risktaker as agent of others who will not know and cannot be consulted. This entails guidance in the exercise of administrative discretion, and the remarks will focus on the possibility of finding such guidance. (In effect, in these circumstances, the challenge is to find indicators that point to the public interest rather than the public servant's interest.)

This is, in a sense, a cautionary tale: In our enthusiasm for finding and promoting the innovative, daring, imaginative, decisive and venturesome public servant as entrepreneur, in the model of the swashbuckling CEO, perhaps we should not forget that the responsible public servant also is dealing out risks to life and limb for countless others who don't even know they are in the game.

The theme is both intriguing and challenging. At first glance, it seems that the public service at all levels could do with more risk-takers--managers with, imagination and 
confidence and resolve, ready to propose or back new ideas and willing to risk ruin (or at least exposure) by trying them. How we can encourage those kinds of attitudes and that kind of behaviour? In our public institutions how can we create an environment that is conducive to more nerve, and less nerves, how we can facilitate more risk-taking behaviour in our government offices and town halls, in our universities, in our hospitals, in our prisons and parole systems, in the Pentagon? Suddenly the issue doesn't seem quite as straightforward, perhaps. One might note that we are not talking here about taking risks with the public--the government's job is to protect the public from risk after all. We are considering individual risk-taking, and people who, in spite of an uncertain future, are nevertheless willing to take tough decisions and bear the consequences if they turn out badly.

But the problem is that individual risk-taking in the context of the kinds of business in which the government is involved is likely to have collective consequences. In some decisions that are internal, procedural, organizational, the risks are essentially personal. They may be personal both in representing a risky step for the individual making the decision, and in the fact that their burdens or costs focus narrowly on a few public servants, not on the "public" more generally. Examples include dismissals, downsizing, or decentralization. Clearly, in some of these circumstances, one would like to encourage decisive, innovative, venturesome individual decisions. One would like people willing to exercise their judgment, act on their initiative without having to gather round them all the committees and protocols which, in effect, "sell insurance" by diffusing responsibility. And yet, we have to leash our enthusiasm a bit. The spectre of the apparatus of the state in the hands of people serving the higher calling of their own convictions without restraints, without protocols, without accountability, is a little chilling. Moreover, in areas of public policy as diverse as public health, waste management, product safety, transportation of goods, criminal justice, fire protection, the location of chemical plants, defense and foreign policy, most of us would want to be assured that, in uncertain conditions, decision taking by our proxies in public agencies is based on the best procedures available for decision making in such conditions. This paper considers briefly aspects of risk taking by public managers including questions of why public servants won't take risks, and why perhaps they shouldn't.

\section{"Risk Taking in the Public Service"}

For a variety of reasons, risk-taking behaviour of the "sticking your neck out" kind is apparently in short supply in the public service--people apparently won't take risks even when they should. Individual attitudes and behaviour are conditioned by and reflect the decision culture of the organization. Not only does the decision culture vary from institution to institution, but even within organizations. In Canada over the last two decades (with the traditional lag a similar evolution probably occurred here earlier) there has been a significant turnaround in the prevailing mood within government as to its role in economic developments. The pendulum has swung from the optimism of the 1960 s reflecting positive expectations about state intervention to the pessimism of the 1980s regarding the pernicious consequences of misguided government efforts to offset market imperfections. The 1960s were a period of economic buoyancy--the time when almost all the major 
national entitlement programs were introduced or significantly expanded; the time when bold new program initiatives could be proposed through PPBS (the system of Planning, Program, Budgeting), when comprehensive rational planning and ex ante evaluation and analysis were widely accepted as powerful decision tools.

By the mid-70s, of course, deficits and inflation had taken the bloom off that particular rose. In Canada this became the era of the Auditor General, when attention and analysis shifted to audit and ex post evaluation, to a new kind of comprehensive or value for money auditing that went beyond issues of probity and prudence to report on economy, efficiency and effectiveness. With analysis pressed into service for the processes of internal and external evaluation, the public servant's decisions were increasingly subject to a regime in which even small failures were held up to public criticism and there was no corresponding reward for successes. Consequently, the public servant's environment is highly intolerant of error and conducive to the extreme avoidance of risk. The process of scapegoating, which seems to have become a feature of accident inquiries, investigations and auditors' reports, has pushed too far in the direction of repressing both innovation and a responsible acceptance of risk.

There are, of course, many other examples of systemic pressures leading to strong biases against accepting reasonable risks. Increasinglymedical practitioners observe that they are forced to do everything technologically possible to preserve or prolong the lives of every case of damaged fetus or terminally ill patient, simply because of the ex post liability imposed through the court system and malpractice or "wrongfullife" type litigation. Clearly, such a system forces abdication of individual responsibility and discretion. It is also clear that collectively the system is ultimately unsustainable; but the individual doctor can't take the risk of discretionary action that would not be considered procedurally impeccable in some retrospective scrutiny.

With the move to restraint or cutback management in the late 70 s and early 80 s, the swing was almost complete. Admittedly, contraction and retrenchmentdon't exclude imaginative initiatives and bold new measures. Risks can be taken in the course of downsizing and privatization, no doubt, even though by progressively fewer people. But most would concede that the current round is hardly one that encouragesindividual risktaking. And it would be naive to expect any significant change in the personal risk-taking behaviour of public managers without some corresponding prior change in the decision culture within public institutions, and particularly without some change that will bring the rewards and penalties attached to such behaviour more into line with practice in the private sector. This presents a tough challenge.

One aspect of this challenge simply may be a communications problem. Work by David Zussman (1988) suggests that the most dramatic difference between the attitudes of managers in the public and private sectors is the confidence with which middle and lower level managers express a grasp of the organization's goals and objectives. In the private sector the goals expressed by leadership are clearly felt by all management. In the public sector, top management expresses confidence that they know the organization's purposes and objectives, but the level of confidence falls uniformly and 
dramatically as one moves down the organizational hierarchy. Evidently if you feel confident about objectives--and about being judged on attainment of objectives--you are likely to feel prepared to exercise more discretion about how you get there. If you know only the rules, not the direction they will lead, then you play by the rules, rather than exercise imagination.

Zussman's work also suggests that, in the Canadian context at least, the traditional public service emphasis on probity and value for money has had an impact. In the private sector, top managementand lowerlevel managementalike respond by listing the "importance of human resources" and "excellence-competence" as values encouraged among managers, while in the public sector, management lists "efficiency-effectiveness" and "value for money" as the top-ranking priorities. In the private sector, more than $50 \%$ of managers described their organization as oriented toward innovation and creative management; in the public sector, only about $25 \%$ characterized their departments that way. Interestingly, management in neither sector had a strong perception that risk-taking was rewarded. Fewer than $20 \%$ in the private sector believed that to a great or very great extent risk-taking was rewarded in their organizations; in the public sector this number fell below 5\%. In both sectors, the number decreases as the number of levels from the DM/CEO position increases.

Although this is generalizationbuilt on a single large 1986 sample, it is suggestive. The sampling was repeated in the summer of 1988, and further results should be available in late 1989.

In his recent book, Beyond the Bottom Line: Management in Government, Tim Plumptre (1988) has explored why the organizational culture in government does-and may inevitably-differ from that in the private sector.

Introducing comparisons with the private sector can be trite. As Wallace Sayre noted, the public and private sectors are alike in all unimportant respects. In fact, of course, the private sector is no more homogeneous than the public sector and the organizations that inhabit it do not uniformly exhibit the characteristics of entrepreneurism, efficiency and flexibility that are supposed to be the requisites for survival. Management in the public sector- certainly at its senior levels-is different from, and often harder than management of the private organization. The senior official must attempt to satisfy many more constituencies than the typical CEO and must manage organizations whose objectives are poorly defined and whose authority structure is cluttered and convoluted. Making decisions, particularly those involving risk, within a context that imposes extensive requirements for consultation and that provides less privacy, less control over resources, and less flexibility to motivate and reward, clearly represents more than simply a difference of degree.

The fundamental point is that the impediments constraining risk taking by: the public manager exist precisely because of the business of government. In the event of failure, individual risk taking in economic ventures on the part of the CEO can have negative consequences for the risk taker and serious consequences of a financial nature for shareholders, employees and clients. But when a city official decides whether to install new street lighting, a highways official chooses how to spend a limited budget, a parole officer decides the status of a released prisoner, an environmental official chooses how 
forcefully to act against a factory violating emissions standards, or when a state official considers whether to permit herbicide spraying, all are making decisions with direct consequences for public risk, risks to individuals who have never had an opportunity to consent in a voluntary and informed way even to the extent that is available to a shareholder.

In British Columbia, a whole new tradition of "sympathetic administration" has developed recently. This new brand of administrative justice, or new emphasis in the exercise of administrative discretion, involves ignoring regulations and laws on waste in forest management (because times were tough for the timber industry), overlooking violations of emission standards, and pollution controls by pulp mills and other wood products operations (because, though extensive, they were "minor"), and issuing mining exploration licenses in old and new provincial and national parks (because it would be too expensive to buyout the mineral rights and they won't find anything (anyway). The risks to future generations in these entrepreneurial liberties are not discussed or taken into account in any way, so far as one can tell. But they should be.

Public managers routinely make decisions in all these areas, both as advisers to elected officials and as administrators of programs and policies. Individual administrators exercise to achieve goals reflecting the collective preferences revealed through the machinery of democracy. The public manager is called upon to take risks as a proxy or agent for the public interest. For decision taking of this kind, what options are available for responsible decision-making? Presumably it must be more than the machinery of Bayesian decision analysis developed to enable individuals to bring their own personal preferences and attitudes toward risk to bear on choices under uncertainty.

The standard technique advocated for public policy decisions involving risk is variously known as Risk/Benefit Analysis--RBA--or Quantitative Risk Assessment. In the prevailing mood of skepticism towards the usefulness of ex ante evaluation, RBA has received its share of criticism and disdain. As its name suggests, RBA is derived from Cost/Benefit Analysis but takes it a step further by introducing probabilistic estimates of the risks of consequencesin order to arrive at some expected net present value for a decision.

Both the probabilities and the consequences in an RBA must usually be estimated and the precision of these estimates will vary widely from problem to problem. Thus in areas such as highway safety, often one can state with high confidence that a particular improvement will save a certain number of lives per year on average. Much less precise estimates of probabilities are available when considering new technologies, such as the siting of nuclear power plants or liquid natural gas reservoirs, which may present very small probabilities of catastrophic losses.

Similar difficulties occur when estimating the possibly distant consequences of rare events. For common events such as automobile accidents or fires, for instance, historical data can be used to estimate loss of life, injury and property damage. But the long term consequences of exposure to toxic or carcinogenic substances are much more difficult to anticipate since the information available from epidemiological studies or animal bioassay studies is often flawed in vital respects. With issues like acid rain, the ozone layer, greenhouse gases, or global climate change and risks to national security- the prospects 
for good estimates of either probabilities or consequences obviously are poor. But even here the issue must be faced.

Nevertheless, when the estimates have been chosen, an RBA adds all the risks so derived and combines them with the known or estimated costs and benefits of the decision to measure the overall net social benefit. As with Cost-Benefit Analysis, this full treatment requires that all costs, risks, and benefits be expressed in a common measure, usually Net Present Value in dollars.

Without going into detail, one can note several referencesto methodology (Crouch \& Wilson, 1982; Hertz \& Thomas, 1983) and several to an outstanding ethical dilemma that arises for those electing to make risky decisions on behalf of others. The way people seem individually to perceive and balance risks (Kahneman \& Tversky, 1979) do not seem to fit the rules on how you ought rationally to gamble when you must (Raiffa, 1968), which presumably are the rules that should guide the public administrator conducting a proper risk-benefit analysis.

RBA as a decision-making technique has been criticized on other grounds as well. This paper will consider the possibility that it is in fact simply morally unacceptable-that it represents an infringement on rights that are basic to a culture-perhaps particularly to American culture. But first, it examines analytic difficulties. Extreme scientific uncertainty prevails on many of the most important questions related to currentpublic debates on risk. There is a danger that reliance on numbers will exaggerate the clarity or certainty of the scientific facts relevant to a decision, and this effect can be manipulated to point overwhelmingly toward only one possible decision. This is the "numbers made me do it" escape clause. Also, the requirement that quantities be expressed in monetary values has led critics to charge that estimates of these quantities are subjective and any resultant calculation of total risk will be completely arbitrary.

Critics also argue that this type of analysis neglects distributive issues. Risk controversies typically pit one social group against another--the conflict is most often between commercial interests wishing to engage in risky activities for profit, and consumer, labour or community groups opposing the activity because they will bear the risk. The ethical basis of RBA is utilitarian, and any mechanical application of the techniques will not account for the fact that a particular distribution of risks, costs and benefits might be morally or politically unacceptable.

A third objection is that RBA requires that costs, risks and benefits be expressed on a common scale so that quantities that are incommensurable can somehow be compared. This involves putting prices on the priceless-- one's home, friends, family, the beauty of an unspoiled environment, and human life itself. According to this argument, although many collective decisions, such as highway improvements or the provision of rescue facilities, can retrospectively be determined to imply a particular value of human life, collective discretion based on the conscious awareness of this valuation must not be exercised in advance of a decision. In other words, it seems that decisions that result in injury or death can be tolerated socially as long as the consequences are not explicitly considered in making the decision. 
Some, but not all, of these criticisms have validity. What are the alternatives for decision making in the area of risk?

One approach is to abandon analysis altogether and rely instead on polls or referenda. This option--"the people made me do it"--seems to lean toward direct democracy and consequently has great populist charm. It also has major weaknesses. First, it wastes the advantages of representative democracy. Representatives are selected or appointed to devote more time and thought to public policy issues than the average citizen can generally afford. The representative who refers every significant decision back to the constituents is hardly fulfilling his or her responsibility. Furthermore, the resulting decisions are likely to be simply inferior-morally, technologically, or economically. A majority of the populace, under duress or under the influence of some charismatic individual, might momentarilyendorse a decision that is clearlyunacceptable to the basic values of the country, such as one that violates the rights of a minority group. Polls are so volatile and so subject to influence by advertising, the media, and conspicuous but anomalous events that a policy based on them would be liable to undergo major changes from week to week. And particularly on matters of risk, abundant experimental evidence suggests that people's uninformed choices are subject to major biases and inconsistencies and that, when confronted with the inconsistencies in their naive choices, people tend to change their views to correspond more closely with those of a rational Bayesian decision-maker.

At another point, national culture might influence procedures for risk-taking in determining or implementing public policy. If it is true that the United States is more oriented toward participatory democracy, while Canada tends toward representative democracy, in Canada we might expect to see heavier reliance on experts and bureaucratic structures than on the hearings, consultations, and public input that seem dominant in the United States.

This second approach, to refer decisions involving risk to the consensual, intuitive judgement of a community of experts who will blend technical expertise, business acumen, and common sense, has much to commend it. This approach, "the experts made me to it," is particularly popular when the decision at hand resembles those made within a traditional professional practice such as engineering or medicine. When an established body of professional knowledge exists, expert judgment can yield practical, successful decisions even on matters where scientific certainty is not strictly available.

This is usually accomplished through robust rules of thumb that reflect abundant operating experience and include hefty margins of safety, as in the design of physical structures such as bridges.

This process has two major disadvantages. First, most current contentious risk issues involve new technologies, new chemicals, or new environmental impacts, so operating experience is insufficient to develop reliable informal professionalstandards. Consequently, when experts exercise their judgment, they probably are making guesstimates almost as 
arbitrary as those of anyone else. Second, the process of expert judgment is stubbornly non-transparent. In estimating total risk or recommending a decision, the expert must make a number of assumptions. If professional knowledge does not grant a special authority to the expert's assumptions, then political decision makers will want to know what assumptions were made, and their possible impact on the total estimated risk. But, if the assumptions are made implicitly and rolled together in the expert's head, they are unavailable for scrutiny or for testing of effect.

Thus "the experts have spoken" is likely to prove to be a cop-out that will not fly (to mix the metaphor rather badly). Substantial arguments, however, have been posed in favour of the so-called "science court" or "fact forum" approach to handling certain issues of conflict in interpretation of scientific evidence.

A third common approach is to stick with precedent and the way things have always been done ("past practice supports me"). Any observer of organizational decisionmaking, private or public, will see that in the absence of compelling pressures to the contrary, decisions are made on the basis of precedent, habit, and standard operating procedures. In the realm of public decisions on risk, this approach is exemplified in announcements that justify the imposition of a given risk by comparing it to other risks that are currently imposed and consequently presumed to be acceptable. You might, for example, hear that the risk from a certain source of radiation will be less than that which you face every day from your luminous watch dial. Such comparisons, even if true, may be misleading; does anyone really know the consequences of exposure to luminouswatch dials? While any canny administrator knows that major new approaches to policy are most difficult to push through the organization, ease of implementation is surely the only defense that one could muster for this approach. Such an approach probably won't upset anyone- until something goes wrong. But when traditional standards, practices, and techniques are applied to new decision problems, something is bound to go wrong sometime, simply because the environment has changed. This approach has neither rationality, consistency, equity, nor scrutability to commend it.

Thus, I've introduced the notion of risk-benefitanalysis as a natural extension of wellknown procedures for cost-benefit analysis undertaken under conditions of uncertainty, in the face of risk. I've also referred to the underlying methodology; noted a few obvious criticisms of that analytical approach, and considered some possible alternatives to it. All were found wanting in some key respects, which forces us back to the central role of analysis and the analyst in the public service.

Where does this leave us with respect to this issue of risk-taking by the public manager? After retracing briefly the logic of what I have been arguing, the paper concludes with observations that might serve as a starting point for further discussion.

Risk-taking of the kind we all agree is needed--the imaginative and creative behaviour that is the essence of innovation --will only occur in an environment where individual administrative discretion is not too rigidly constrained and in a decision culture that tolerates some negative outcomes in the cause of making progress overall. The 
problem is that risk-taking decisions by the public servant will inevitably lead, in many instances, to taking risks with the public. In these situations, what guidelines can point us in the direction of responsible risk-taking?

One prominent school of thought has argued that decision making of the rational analytic kind outlined earlier is based on a false ethic--namely that some social or public welfare exists that can be quantified and that takes precedence over the welfare of individual members of the collectivity. These critics maintain that the individual is paramount and that the over-riding value of the human life that is at the core of all our social institutions means that public servants should base all proxy decision making on what they term a deontologicalethic, rather than a utilitarian ethic. All decisions involving risk must be made by balancing the rights of individuals, with rights to life and safety taking precedence over pragmatic considerations such as economic benefits, no matter how widely distributed (Dworkin, 1981; Kluge, 1987). The public servant should never be permitted to make decisions that will subject some or all members of the public to involuntary risk.

No doubt, this is an important argument, central both philosophically and practically. But I would argue that it simply sets the framework of principle within which the rational calculations just described must be undertaken. When informed consent cannot practically be obtained from all concerned, the public servant has a responsibility to infer, where possible, the actions and the exchanges of rights in which individuals would be prepared to engage voluntarily. A variety of procedures are available to assist this process within a risk-benefit framework; some are sketched by Robin Gregory in a series of papers and the references he cites (Gregory, 1987, Gregory \& Mendelsohn, 1987) .

We are not concerned with the public servant as God, but with the public servant as agent, or proxy.

This dilemma echoes a lively debate that goes back more than 50 years and that is associated particularly with the writings of two academicians, Carl Friedrich and Herman Finer. Friedrich argued that control over administrative discretion could only be partial and incomplete, and that the best approach was to work toward an improved sense of subjective responsibility to internalized ideals and standards. Accountability, in his view, could never be imposed, only elicited. Finer, on the other side, insisted that society could not rely on the self-directing capacities of the public service and that technical knowledge and creative ideas should be held in check by formal institutional devices. Each side can point to excesses that arise from the other's recommendation.

In the end it still remains that in small decisions as well as large the public manager will ultimately face the existential fact of being condemned to choose. In that choice one has conscience, certainly, and one also has the responsibility to seek informed consent by laying out the consequences of each decision. Risk analysis is vulnerable to profound uncertainty, but so is every other approach. It requires the pricing of the priceless and placing, monetary values on human life, but every other approach does so implicitly. Analysis can be hijacked to serve the interests of power constituencies, but so can expert judgement, staying with precedent, and even the polls (through) ingenious timing or 
cunning manipulation of the media). Although analysis does not initially address the distribution of risks, it can be made to elucidate them and, through techniques of contingent evaluation, it can incorporate procedures for ex ante compensation or liability insurance. Even in a world of profound uncertainty and missing data, explicit and formal analysis offers the essential decision tool for informing and guiding the public manager in the often agonizingly difficult decisions that must be taken on matters of public risk. Within a framework of principle, analysis does help.

\section{References}

Dworkin, R. (1981 ). Principle, policy, and procedure. In R. Dworkin (Ed.) Crime, proof, and punishment, essays in memory of Sir Rupert Cross. London and Boston: Butterworths.

Gregory, R. (1987, December). Risk communication as a tool for improving risk evaluation. Paper presented to the Risk Communications Symposium, Ottawa, Canada.

Gregory, R., \& Mendelsohn, R. (1987). Integrated risk valuation. Manuscript submitted for publication.

Kahneman, D., \& Tversky, A. (1979). Prospect theory: An analysis of decisions under uncertainty, Econometrica, 42(2), 263.

Kluge, E., \& Henner, W. (1987). What is a human life worth? In J. W. Langord (Ed.), Fear and ferment: Public sector management today. Halifax: Institute for Research on Public Policy.

Nagel, T. (1978). Ruthlessness in public life. In S. Hampshire (Ed.), Public and private morality. Cambridge: U.P.

Plumptre, T. W. (1988). Beyond the bottom line, Halifax: Institute for Research on Public Policy. Raiffa, H. (1968). Decision analysis. Reading, MA: Addison-Wesley.

Zussman, D., \& Jabes, J. (1988). Survey of managerial attitudes: Preliminary findings. Institute for Research on Public Policy Working Paper. 